\title{
Tuberculosis infantil en Arica
}

Drs. Milton González A., ${ }^{*}$ Marco A. Aguirre B., Biffret Dłaz A.," Srta. María Avalos O.,"* Sra. Hilda Ponce A.***

\section{ABSTRACT}

\begin{abstract}
A total of 68 children with tuberculosis were analized and controlled in the infantile Bronchopulmonary Unit of the Dr. Juan Noé Hospital of Arica, during one year. from January lrst to December 31rst of 1979. Clinical, Epidemiologic, Radiologic and Bacteriologic aspect were evaluated. Tubenculosis is a problem in jural Aynará population, and it is convenient to improve BCC vaccination and tubertulin testig control.
\end{abstract}

En las últimas décadas, debido a los avances epidemiológicos y quimioterápicos, la tuberculosis en Chile, al igual que en el resto del mundo, ha disminuido progresivamente como causa importante de muerte; ${ }^{1-5} \sin$ embargo, la incidencia, aunque también ha experimentado un descenso, continúa siendo elevada, ${ }^{1,4,7,9}$ y es así como en el año 1979 alcanzó cifras de $49,5 \times 100.000$ habitantes, en los menores de 15 años en esta provincia. ${ }^{9}$

Arica presenta una de las más altas prevalencias de tuberculosis en el país, ${ }^{7,9}$ hecho favorecido por ser una ciudad limítrofe, con una población urbana que desarrolla un constante comercio entre los países vecinos. Por otsa parte, su población nural. aunque proporcionalmente pequeña $(5,5 \%),{ }^{10}$ es de origen aymará en una gran mayoría, y se encuentra esparcida en el altiplano en pequeños caseríos, desarrollando fundamentalmente la actividad de pastores nómadas.

Estas características, tan propias de la provincia de Arica, dificultan la ejecución de los programas antituberculosos; es por ello que se decidio efectuar un análisis de los casos de tuberculosis infantil, con elobjetivode actualizar nuestro conocimiento de la realidad de esta patología en la zona.

\section{MATERLAL Y METODO}

Se analizan retrospectivamente 68 niños tuberculosos, diagnosticados en la Policlínica Broncopulmonar del Hospital Dr. Juan Noé, de Arica, entre el $1 .^{\circ}$ de enero y el 31 de diciembre de 1979.

*M édico Pediatra Hospital Juan Noé, de Arica.

**Enfemera Lnidad Broncopulmonar Hospital Juan Noe, de Arica.

***Asistente Social Unidad Broncopulmonar Hospital Juan Noé, de Arica.
Fueron considerados los siguientes parámetros:

a) Edad; b) Sexo; c) Estado nutritivo, según relación peso-edad, por Tabla de Sempé; d) Residencia: se consideró sector urbano o rural según delimitaciones comunales respectivas; e) Estudio socioeconómico, según ficha de estratificación social del Ministerio del Interior; $f$ ) Antecedentes de Contacto, considerando como tal aquel que cohabita con el paciente; g) Síntomas y signos que orientaron el diagnóstico; h) Localización: se clasíficó en tuberculosis inaparente, pulmonar y extrapulmonar, según criterio de normas sobre TBC Infantil, Chile 1974; i) PPD realizado según técnica de Mantoux, considerándose positivo aquel mayor $6 \mathrm{~mm}$ en el menor de 6 años y mayor a $8 \mathrm{~mm}$ en los niños mayores; j) BCG, se consideró vacunado aque] paciente que presentaba cicatriz; k) Radiología: clasificación de lesión y extensión según normas de TBC. Infantil, Chile 1974; 1) Bacteriología: técnica de Zielhn-Nielsen para baciloscopias y técnicas de Lewestein-Jensen para cultivo efectuadas por tecnólogo médico en el Laboratorio de Bacteriología de Koch de nuestro hospital.

\section{RESULTADOS}

De los 68 niños analizados podemos destacar:

En relación a los grupos etáreos se comprueba que la mayor ocurrencia de TBC Infantil se produce en el grupo escolar, $56 \%$ (38 niños), seguido por el grupo preescolar, $29 \%$ ( 20 niños), siendo el grupo de lactantes el de menor ocurrencia, $15 \%$ (10 niños).

Cabe destacar que la distribución etárea de los pacientes controlados es coincidente a la distribución etérea de la población infantil ariqueña.

En el grupo de lactantes se encuentran sólo 2 casos (2,9\%) (Gráfico N. $\left.{ }^{\circ} 1\right)$. 


\section{Gráfico N. I}

Hospital Juan Noé

Arica

TBC Infantil

Distribución etárea

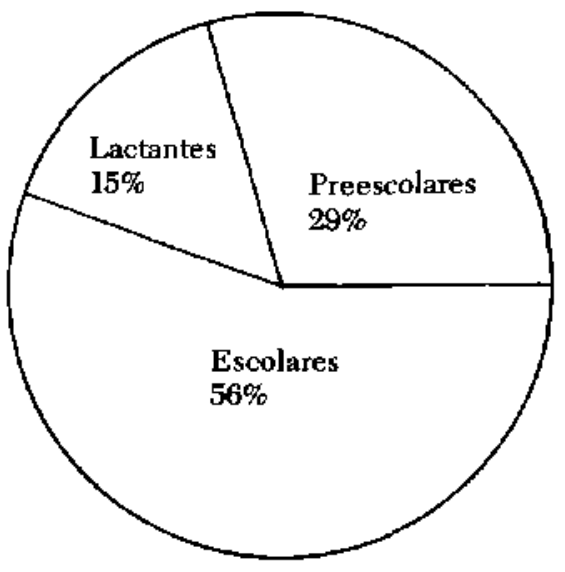

En referencia a la distribución por sexo, no se encuentran diferencias significativas en el grupo estudiado, correspondiendo un 51,5\% (35 niños) al sexo masculino y un $48,5 \%$ (33 niños) al sexo femenino (Gráfico N. ${ }^{\circ}$ ).

\section{Gráfico N. 2}

TBC Infantil Distribución por sexo

Hospital Juan Noé Arica

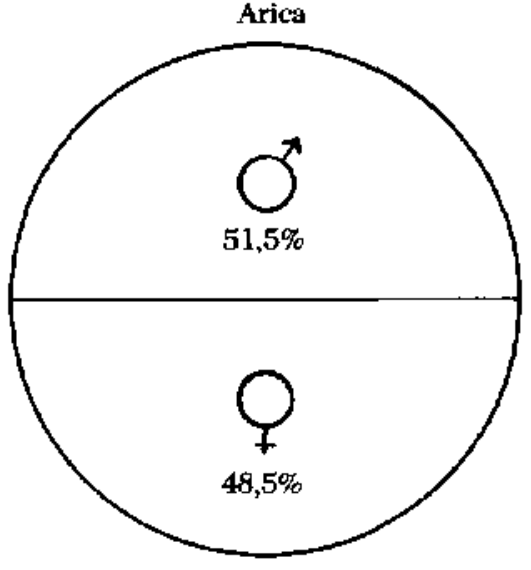

El estudio del estado nutritivo de los pacientes demuestra que el 4,4\% ( 3 niños) tienen sobrepeso, el $72 \%$ ( 49 niños) son eutróficos y el $23,5 \%$ ( 16 niños) son desnutridos.

En el grupo de nínos desnutridos se comprueba
Distribución población ariqueña

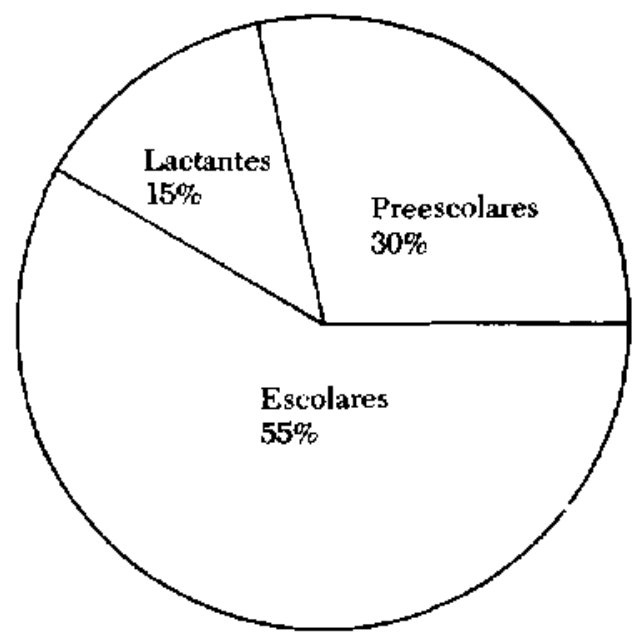

que el 56,2\% (9 niños) muestran signos de desnutrición leve, el 18,8\% (3 niños) son portadores de desnutrición grave.

Es necesario insistir que en el momento del diagnóstico el 76,4\% de los niños tienen un estado nutritivo de eutrofia o sobrepeso y sólo un $23,5 \%$ presentan algún grado de desnutrición.

En referencia a los casos de desnutrición, se observa que la distribución en sus tres diferentes grados no es concordante con la distribución de ésta en la población ariqueña, encontrándose que en los niños tuberculosos existe un aumento de casos moderados y graves $(43,8 \%$ ) (Tablas 1 y 2 ).

Tabla 1

Distribución por estado nutritivo

Hospital Juan Noé

Arica

\begin{tabular}{lcc}
\hline ESTADO NUTRITIVO & N. & $\%$ \\
\hline Sobrepeso & 3 & 4,4 \\
\hline Eutrófico & 49 & 72,1 \\
\hline Desnutrido & 16 & 23,5 \\
\hline Total & 68 & 100,0 \\
\hline
\end{tabular}


Tabla 2

TBC Infanti]

Distribución en grados de desnutrición

Hospital Juan Noé

Arica

\begin{tabular}{lcc}
\hline $\begin{array}{l}\text { GRADO DE } \\
\text { DESNUTRICION }\end{array}$ & N. $^{\circ}$ & $\%$ \\
\hline D. leve & 9 & 56,2 \\
\hline D. moderada & 3 & 18,8 \\
\hline D. grave & 4 & 25,0 \\
\hline Total & 16 & 100,0 \\
\hline
\end{tabular}

En relación al lugar de residencia de los pacientes controlados, se observa que un $30,9 \%$ (21 niños) corresponden al sector rural, y en un $69,1 \%$ (47 niños) al sector urbano de Arica.

Esta distribución no es concordante con la ruralidad de la provincia de Arica, considerada aproximadamente en un $\mathbf{5 , 5 \%}$.

Es importante destacar, además, que étnicamente la gran masa poblacional rural de la provincia es de ascendencia aymará (Gráfico N. ${ }^{\circ} 3$ ).

\section{Gráfico N. ${ }^{\circ} \mathbf{3}$}

TBC Infantil

Distribución seguin lugares de residencia

Hospital Juan Noé

Arica

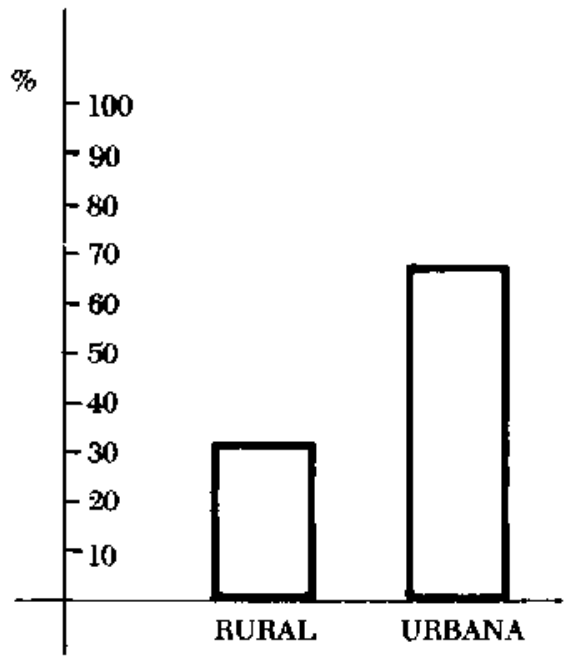

\section{Estudio socioeconómico}

Con respecto a la situación socioeconómica, destaca que en un $86 \%$ de los pacientes ésta es regular o mala y en sólo el $14 \%$ de los niños ésta es buena (Tabla 3).

Tabla 3

TBC Infantil

Condición socioecconómica

Hospital Juan Noé

Arica

\begin{tabular}{lcc}
\hline CONDICION SOCIOECONOMICA & N. ${ }^{\circ}$ & $\%$ \\
\hline Buena & 9 & 14 \\
\hline Regular & 35 & 51 \\
\hline Mala & 24 & 35 \\
\hline Total & 68 & 100 \\
\hline
\end{tabular}

Al revisar la anannesis de los pacientes se encuentra el antecedente de contacto tuberculoso en el 72\% de ellos (Tabla 4).

Table 4

TBC Infantil

Antecedente de contacto TBC

Hospital Juan Noé

Arica

\begin{tabular}{lcc}
\hline $\begin{array}{l}\text { ANTECEDENTE } \\
\text { CONTACTO }\end{array}$ & N. & $\%$ \\
\hline Si & 49 & 72 \\
\hline No & 19 & 28 \\
\hline Total & 68 & 100 \\
\hline
\end{tabular}

Haciendo un estudio de la sintomatologia se encuentra que el $40 \%$ de los pacientes ( 27 niños) son asintomáticos y el resto, $60 \%$ (41 niños), son sintomáticos. De estos 41 niños, un $66 \%$ presentan síntomas generales tales como anorexia, fiebre, baja de peso y decaimiento, un $39,8 \%$ presentan síntomas respiratorios tales corno tos, expectoración y hemoptisis. Otros síntomas de 
menor relevancia se refieren a localizaciones extrapulmonares específicas (Gráfico N. ${ }^{\circ}$ ).

\section{Gráfico N. ${ }^{\circ} 4$ \\ TBC Infantil}

Sintomatologa

Hospital Juan Noé

Arica

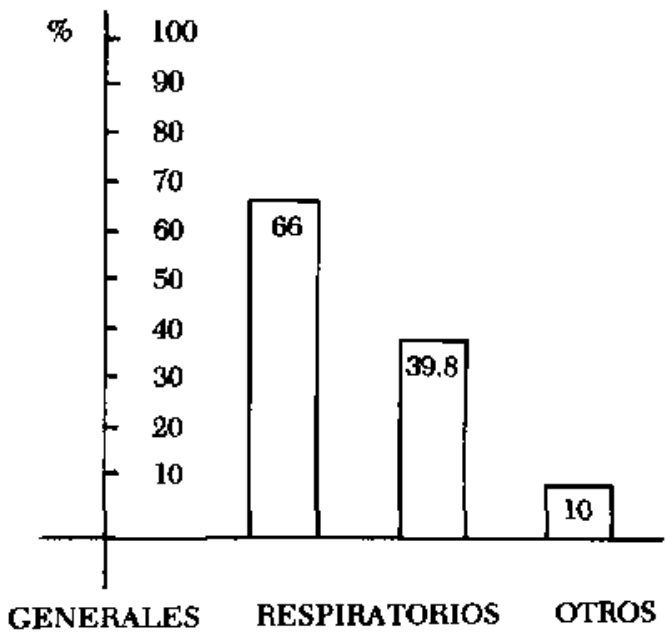

En el $54,4 \%$ de los niños el examen físico fue negativo y en un $45,6 \%$ de los casos se encontró algún tipo de signología (Gráfico N. ${ }^{\circ} 5$ ).

\section{Gräfico N. ${ }^{\circ 5}$}

TBC Infantil

Examen físico

Hospital Juan Noé Arica

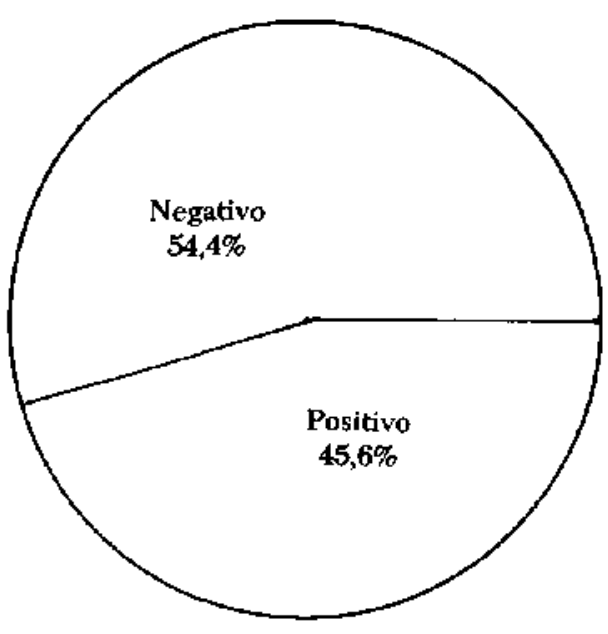

En relación a la localización de la tuberculosis, cabe señalar que el 70,6\% (48 niños) presenta TBC con localización pulmonar, observándose en 3 de ellos una diseminación miliar.

Referente a los casos de TBC extrapulmonar, se encontró un caso cuya localización fue meningea (Tabla 5).

\section{Tabla 5}

TBC Infantil

Localización

\begin{tabular}{lcc}
\hline LOCALIZACION & $\mathrm{N}^{\circ}$ & $\%$ \\
\hline Inaparente & 12 & 17,6 \\
\hline Pulmonar & 48 & 70,6 \\
\hline Extrapulmonar & 8 & 11,8 \\
\hline Total & 68 & 100,0
\end{tabular}

En referencia a la prueba de la tuberculina deberá considerarse que:

En el 20,5\% (14 pacientes) la prueba no se realizo por: a) Desabastecimiento del producto y b) Por considerarse innecesaria en razón de haber una confirmación bacteriológica previa.

De los restantes 54 pacientes a los cuales se les practicó el PPD, se observaron los siguientes resultados: el 20,4\% (11 niños) con PPD (-) negativo y el 75,9\% (41 niños) con PPD (+) positivo.

En un 3,7\% (2 niños) se carece de información por irregularidad en la concurrencia de los pacientes a lectura en la fecha de citación (Tabla 6).

\begin{tabular}{|c|c|c|}
\hline & & \\
\hline PPD & N. ${ }^{\circ}$ & $\%$ \\
\hline Positivo & 41 & 75,9 \\
\hline Negativo & 11 & 20,4 \\
\hline Sin información & 2 & 3.7 \\
\hline Total & 54 & 100,0 \\
\hline
\end{tabular}


El $86 \%$ de los pacientes estudiados presen. taban cicatriz BCG y el $14 \%$ no la presentaba (Tabla 7).

Tabla 7

TBC Infantil

BGC

Hospital Juan Noé

Arica

\begin{tabular}{|c|c|c|}
\hline BCG & No & $\%$ \\
\hline Con cicatriz & 58 & 86 \\
\hline Sin cicatriz & 10 & 14 \\
\hline Total & 68 & 100 \\
\hline
\end{tabular}

En cuanto a la extensión radiológica predominan las formas inaparentes $(17,6 \%)$ y mínima $(60,3 \%)$ (Tabla 8 ).

Tabla 8

TBC Infanti]

Extensión radiológica

Hospital Juan Noé

Arica

\begin{tabular}{lcc}
\hline EXTENSION & N. & $\%$ \\
RADIOLOGICA & 12 & 17,6 \\
\hline Inaparente & 41 & 60,3 \\
\hline Minima & 11 & 16,2 \\
\hline Moderada & 4 & 5,9 \\
\hline Avanzada & 68 & 100,0 \\
\hline Total & & \\
\hline
\end{tabular}

\section{Lesiones radiológicas pulmonares}

En los 68 niños estudiados predominaron como hallazgo radiológico: La normalidad $(29,4 \%)$, luego la persistencia radiológica infiltrativa hiliar y perihiliar más de 2 meses (25\%) y la asociación gangliopulmonar (22\%) (Tabla 9 ).

\section{Table 9}

TBC Intantil

Lesiones radiologicas pulmonares

Hospital Juan Noé

Arica

\begin{tabular}{lcc}
\hline \multicolumn{1}{c}{ TIPO DE LESION } & N. & $\%$ \\
\hline Sin lesión radiológica & 20 & 29,4 \\
\hline Infiltración hiliar mayor de 2 meses & 17 & 25,0 \\
\hline $\begin{array}{l}\text { Gangliopulmonar } \\
\text { Sombras nodulares de relleno } \\
\text { alveolar bilateral }\end{array}$ & 15 & 22,1 \\
\hline Pleuresía & 9 & 13,2 \\
\hline Diseminación bilateral & 2 & 2,9 \\
\hline Atelectasia basal & 2 & 2,9 \\
\hline Neumotórax y derrame pleural & 1 & 1,5 \\
\hline Caverna & 1 & 1,5 \\
\hline Total & 68 & 100,0 \\
\hline
\end{tabular}

Con respecto al estudio bacteriológico, éste fue positivo en un $44,1 \%$ de los casos, de éstos en un $14,8 \%$ ( 8 casos) fue por baciloscopía directa y un $32,3 \%$ (22 casos) fue exclusivamente por cultivo de Koch (Tabla 10).

Tabla 10

TBC Infantil

Confirmación bacterióógica

Hospital Juan Yoé Arica

\begin{tabular}{lcccc}
\hline $\begin{array}{l}\text { CONFIAMACION } \\
\text { BACTERIOLOGICA }\end{array}$ & B K & CULTIVO & TOTAL & $\%$ \\
\hline Con confirmación & 8 & 22 & 30 & 44,1 \\
\hline Sin confimación & - & - & 38 & 55,9 \\
\hline \multicolumn{1}{c}{ Total } & 8 & 22 & 68 & 100,0 \\
\hline
\end{tabular}




\section{COMENTARIO}

La tuberculosis infantil se observa a cualquier edad; sin embargo, se demostró un porcentaje mayor en los grupos escolares y preescolares, distribución proporcional a la población infantil de Arica.

Con respecto al sexo, no fueron observadas diferencias significativas.

El estado de nutrición en la inmensa mayoría de los pacientes fue normal ( $72 \%$ ). No obstante, se comprobó una mayor incidencia de desnutrición $(23,5 \%)$ en los niños tuberculosos, en comparación a la observada en la población infantil ariqueña (8\%).

Con respecto a la residencia, predomina la procedencia urbana $(69,1 \%)$. Sin embargo, destaca que la población rural de la provincia es de aproximadamente un $5,5 \%$ y a ella le correspondió un $30,9 \%$ de los niños tuberculosos analizados. Importante es señalar que el sector rural étmicamente es de ascendencia aymará en su gran mayoría y este hecho podría explicar la elevada incidencia tuberculosa, como ocurre en otras poblaciones indígenas, según informan diversas publicaciones nacionales y extranjeras. $1,5,11$

Destaca el bajo nivel socioeconómico de nuestros pacientes.

El 86\% de los niños tuberculosos presentan cicatriz BCG y un $14 \%$ no, por lo que consideramos baja la protección conferida por el BCG.

$\mathrm{El}$ antecedente de contacto tuberculoso fue observado en el $72 \%$ de los casos y se constituyó en uno de los pilares del diagnóstico de la tuberculosis infartil.

Los śntomas y signos estuvieron ausentes en un porcentaje elevado de los casos y cuando estuvieron presentes, éstos fueron inespecíficos.

En relación a la localización, la más frecuente es la pulmonar $(70,6 \%)$, dato que concuerda con la literatura nacional y extranjera. 1,3,4,6. 11. 12

Con respecto a las formas clínicas, existió un claro predominio de las fonmas minimas e inaparentes.

El hallazgo del bacilo de Koch fue comprobado en el $\mathbf{4 4 , 1}$ \% de los casos, cifra más alta que la descrita en las publicaciones nacionales. ${ }^{\prime}, \lambda \mathbf{I}-13$

En cuanto a la prueba de la tuberculina, nos llamó la atención que fue negativa en un $16,1 \%$ de los casos, cifra que consideramos elevada y pensamos que podría explicarse por la carencia de un buen lector, en ausencia de la única enfermera con que cuenta la Unidad.

Lo anterior conlleva a un proceso de evaluación del programa de TBC infantil en Arica, deduciéndose que el enfoque primordial del estudio de la población debe ser llevado fundamentalmente al sector rural y perfeccionar las técnicas y métodos del BCG $y$ PPD.

\section{RESUMEN}

Se analizaron 68 niños tuberculosos controlados en la Unidad Broncopulmonar Infantil del Hospital Dr. Juan Noé, de Arica, entre el $1 .^{\circ}$ de enero y el 31 de diciembre de 1979. Se valoran aspectos epidemiológicos, clínicos, radiológicos y bacteriológicos. Se deduce la relevancia que reviste la TBC en la población rural aymará y la conveniencia de mejorar el cumplimiento del programa de vacunación BCG y el control con prueba de tuberculina.

\section{REFERENCIAS}

${ }^{1}$ Meneghello, J. Pediatra. Tomo 2. Segunda Edición. Pág. 1597, 1978.

2 Yariez, A. Epidemiología de la tuberculosis. Boletín del Hospital San Juan de Dios. pp. 254-269. Septiembre 1971 .

3 Nelson. W.; Vanghan, V, McKay, R. Pediatra. Tomo l. Sexta Edición. Pág. 599, 1974.

4 Kendig, E. Trastomos pulınnmares. Tomo l. Pág. 671, 1977.

${ }^{5}$ Graybouski, S. Epidemiology of Tuherculosis and the role of BCG. Clinies in Chest. Medicine. pp. 175-186. Mayo 1980.

6 Urdaneta, E. Tubereulosis en el niño. Arehivo: Venezolino Pueric y Pediatri 41: 291, 1978.

7 Grinspun, M. Situación epidemiotósica cle la taberculosis en Chile. Boletin del Hospital San Juan de Dios. pp. 27 I-275. Septiembre $19 r 1$.

${ }^{8}$ Kendig, E. Tuberculosis among Children in the United States. 1978. Pediatrics 62: 269, 1978.

9 Flores, F. Evaluación amual programa THD SA. 1979.

10 INE.

11 Gomejo, H.; Girardi. G.; Mateluna, E.; Olicus, A. Tuberculosis infantil. Boletín del Hospital San Juan de Dios. pp. $380-$ 389. Septiembre 1971 .

12 Ibanez, S.; Ross, B.; Ledesma, O.; Diaz, M. Experiencia de 7 anors en TBC pulmonar intantil. Revisión de 323 casos hos pitalizados en el período 1962 1969. Rev. Chilena de Pediatria 44: 319. 1973.

13 Normas de prevención, trataniento y control de las enfermedades respiratorias de la infancia y cuberculosis infantil. Chile, 1974 . 